\title{
Adelbeid von Saldern \\ Permanente Krise? - Stabilität und Instabilität des \\ Herrschaftssystems im deutschen Faschismus
}

In das weitverbreitete Bild über das $»$ Dritte Reich scheint auf den ersten Blick das Thema "permanente Krise « nicht recht zu passen und schon gar nicht, falls mit dem Begriff "permanente Krise « eine Wirtschaftskrise gemeint sein sollte. Eher - so scheint es - könne man von einer Art Wirtschaftswunder sprechen. Gewisse Zahlen scheinen tatsächlich darauf hinzudeuten: 1938/39 überschritt das reale Sozialprodukt den Vorkrisenstand von 1928/29 bereits um annähernd ein Fünftel; gegenüber dem Tiefstand von 1932 hatte es sich sogar mehr als verdoppelt (Petzina, 1977, S. 108). Während in den Vereinigten Staaten 1937/38 die Arbeitslosenquote $20 \%$ und in Großbritannien 10\% betrug, konnte man in Deutschland mit Stolz auf einen leergefegten Arbeitsmarkt hinweisen (ebd.). Wirtschaftlich ging es bergauf. Selbst erfahrene Beobachter ließen sich von der einmaligen Prosperität, vor allem von der hohen Investitionsrate und den vollen Auftragsbüchern beeindrucken.

Wie die Faust aufs Auge wirkt deshalb der in der neueren Forschung zunehmende Trend, den sozio-ökonomischen Krisencharakter des deutschen Faschismus besonders in den späten 30er Jahren zu thematisieren. Die vorläufigen Aussagen dazu weisen eine beträchtliche Variationsbreite auf. Eine besondere Zuspitzung erfahren sie vor allem bei T. Mason (z.B. 1975 b). Er vertrat als einer der ersten Historiker die These, daß der Zeitpunkt (nicht die Ursachen!) des Ausbruches des Zweiten Weltkrieges im Jahre 1939 als eine »Flucht nach vorne« betrachtet werden müsse. Heute steht er mit dieser Auffassung nicht mehr allein da (s.u.). Selbstverständlich war der Wille zur territorialen Expansion bei führenden Kräften in Staat und Gesellschaft von Anfang an, also schon 1933, vorhanden, wobei das Kriegsrisiko bewußt in Kauf genommen wurde. Von der expansionistischen (kriegerischen) Absicht bis hin zur eigentlichen Entfesselung des Krieges lag aus der Perspektive von 1933 gesehen freilich eine nicht näher vorherbestimmbare Zeitspanne. Den genauen Entwicklungsablauf wußte natürlich niemand im voraus. Vor allem war das Verhalten der ausländischen Mächte nicht im voraus exakt kalkulierbar, erst recht nicht das Zusammentreffen von konkreten Ereignissen, Konstellationen und Prozessen im In- und Ausland und der sich daraus für das NS-Regime ergebende Handlungsspielraum.

Im ersten Teil der folgenden Ausführungen stehen in einer Art Querschnittsanalyse die diversen Krisenphänomene vor Ausbruch des Zweiten Weltkrieges, also um 1938/39, im Blickfeld; im zweiten Teil wird in einer Art Längsschnitt gefragt, ob und ggf. inwiefern von einer Krisenpermanenz gesprochen werden kann, während im dritten Teil das Ausmaß des Krisenbewußtseins und die in damaliger Zeit anvisierten Krisenlösungsvorschläge thematisiert werden.

\section{Gesamtkrise 1938/39.}

1. »Meine Herren, die Finanzen sehen sehr kritisch aus« (in: Mason, 1975 b, S. 163), so leitete Göring die erste Sitzung des Reichsverteidigungsrates im November 1938 ein. Die Ent- 
lassung Schachts als Reichsbankpräsident im Januar 1939 war ein sicheres Anzeichen dafür, daß die Staatsfinanzen bereits so aus den Fugen geraten waren, daß selbst ein Mann wie Schacht ein Weiterarbeiten auf dieser Basis abgelehnt hatte. In der Denkschrift des Reichsbankdirektoriums vom 7.1.1938 stand zu lesen:

"Gold oder Devisenreserven sind bei der Reichsbank nicht mehr vorhanden [...] Die Reserven, die aus der Angliederung Österreichs und aus dem Aufruf ausländischer Wertpapiere und inländischer Goldmünzen gebildet waren, sind aufgezehrt« (in: Boelcke, 1978, S. 302).

Das Hauptproblem lag darin, daß man nicht wußte, wie weitere Mittel aufgebracht werden könnten, um die Aufrüstung voranzutreiben (dazu Boelcke, 1975; ders., 1977). Bis 1939 waren ungefähr 60 bis $70 \mathrm{Mrd}$. RM für die Rüstung ausgegeben worden (Hitler behauptete sogar 90 Mrd. RM). 60\% der Staatsausgaben entfielen im Jahre 1938 auf die Rüstung (BagelBohlan, 1973, S. 144). Im Jahre 1938 verwendete man 17\% des Bruttosozialprodukts auf die Rüstung (in den USA 1\%, in Großbritannien 8\%; Carrol, 1968, S. 184). Die Staatsschuld hatte sich in den wenigen Jahren seit 1933 verdreifacht. Das besondere an der Verschuldung lag sowohl im Ausmaß als auch im Tempo und in der Finanzierungsmethode (Mefo-Wechsel). Die Mefo-Wechsel wurden schon damals als »Wechsel auf die zu erobernden Rohstoff- und Absatzgebiete bezeichnet (in: Kadritzke, 1976, S. 202). Die hohe Staatsschuld belastete nicht nur immens das Haushaltsbudget, sondern erschwerte bereits auch die privatwirtschaftlichen Investitionsmöglichkeiten selbst der Rüstungsindustrien, weil auf dem Kapitalmarkt zunehmend Engpässe auftraten. Noch nie hatte eine Nation in "Friedens«-Zeiten in derartigem Umfang Kriegsmaterial produziert (Bagel-Bohlan, 1973, S. 148).

2. Ein zweites sich zuspitzendes Problem lag in der für die Aufrüstung so wesentlichen Rohstoffbeschaffung. Der Vierjahresplan von 1936 konnte trotz unbestreitbarer Erfolge (dazu z.B. Petzina, 1968; Herbst, 1978, S. 363; Milward, 1977, S. 189ff.) das Rohstoffproblem Deutschlands nicht lösen. Zwar war die Ertragssteigerung besonders bei der Pulverund Sprengstoffprodulktion, der Produktion von Mineralöl und von Aluminium sowie die Beschaffung von Buna und Erz beträchtlich. Doch blieb die Auslandsabhängigkeit Deutschlands bei rüstungswichtigen Rohstoffen trotz aller Autarkiebestrebungen noch immer relativ groß (Petzina, 1975, S. 79f.). Zu deren Import brauchte man entsprechende Devisenmengen. Um aber Devisen hereinzubringen, hätten vor allem die Exportanstrengungen forciert werden müssen, was in Anbetracht der Weltmarktsituaiton jedoch äußerst schwierig war. Im Jahre 1938 setzte nämlich eine bedeutsame »Verflauung der Weltkonjunktur« (Dt. Volkswirt, 26.8.1938), eine »fortgeschrittene Depression am Weltmarkt« (ebd., 2.9.1938), eine »neue Weltdepression«, ein.

Der Welthandel war nicht zuletzt von der Entwicklung der US-Wirtschaft abhängig. Die amerikanische Rezession von Ende 1937 erschwerte wieder den deutschen Export. Die deutschen Waren stießen zudem auch in anderen Ländern auf eine neue heftige Konkurrenz (ebd., 11.3.1938). Die Zukunftsaussichten waren für Deutschland entsprechend ungünstig. 1939 konnte man in der Zeitschrift »Wirtschaft und Statistik« lesen:

"Zu einer allgemeinen, tiefgreifenden Bewegung der Wirtschaft in der Welt und des zwischenstaatlichen Warenaustausches liegen bisher nicht die Voraussetzungen vor« (1939, S. 388).

Es war unter diesen Umständen nicht verwunderlich, daß Deutschland im Jahre 1938 eine negative Handelsbilanz aufwies. Nur durch Rückgriffe auf Devisen- und Rohstoffreserven 
konnte die Produktion noch voll aufrechterhalten werden (Petzina, 1968, S. 109ff.). Wie problematisch die Außenhandelslage war, wird nicht zuletzt dadurch erhellt, daß das Deutsche Reich sogar Waffen exportieren mußte, um Devisen zu bekommen, obwohl man doch selbst die Waffen »notwendig« gebraucht hätte (dazu: Mason, 1975 b, S. 164).

3. Ein weiteres zentrales Problem, das sich gerade am Ende der dreißiger Jahre zuspitzte, war der Mangel an Arbeitskräften. Besonders schwer waren die Exportindustrien davon betroffen, weil sie auf die internationale Wettbewerbsfähigkeit angewiesen waren und aus Kostengründen passen mußten, wenn es galt, Arbeiter durch Lohnerhöhungen und größere betriebliche Sozialleistungen und Leistungsanreize von anderen Firmen abzuwerben (dazu: Mason, 1975 a, S. 107). Eine kleine Anekdote verdeutlicht dies plastisch: So ist die Geschichte von einem Bauunternehmer überliefert, der bei Nacht und Nebel seinem Konkurrenten die gesamte Belegschaft im Omnibus entführt hatte (in: Herbst, 1978, S. 377). Infolge des Arbeitskräftemangels ging auch der Übergang der deutschen Industrie zur Schichtarbeit äußerst schleppend voran (Milward, 1977, S. 37, 46, 85; Thomas, 1966, S. 195). Selbst die Rüstungsindustrie fing an, über Arbeitskräftemangel zu klagen, obwohl sie relativ hohe Löhne zahlte.

Das Arbeitskräftereservoir war weitgehend ausgeschöpft. Dies gilt vor allem für die Landarbeiter, die - durch materielle und sonstige Anreize gelockt - schon seit Jahren vom Lande in die Stadt geströmt waren. Diese enorme Landflucht (die Zahl der Landarbeiter war zwischen 1933 und 1938 um 16\% gesunken (Mason, 1975 b, S. 166)) bewirkte ihrerseits wieder einen überaus großen Mangel an Landarbeitern, so daß die sogenannte »Erzeugungsschlacht « in der Landwirtschaft deshalb selbst auch an einen entscheidenden Wendepunkt geriet. Im Jahre 1939 fehlten 250000 Arbeitskräfte in der Landwirtschaft (ebd., S. 167). Auch hier fand deshalb ein gravierender Raubbau an der Arbeitskraft der in der Landwirtschaft verbliebenen Menschen, vor allem der Bäuerinnen, statt (Jacobeit, 1982). ${ }^{1}$ Die Versuche des Staates, Ersatz zu beschaffen, indem Jugendliche - vor allem Mädchen - zur Mithilfe aufs Land geschickt wurden (Landhilfe, Landjahr, Pflichtjahr, Reichsarbeitsdienst etc.), lösten das Problem nicht, denn die Stadtjugend war für solche Arbeiten nur begrenzt brauchbar. Diese Engpässe auf dem Arbeitsmarkt verdichteten sich in rasantenı Tempo, obwohl die Industrie schon seit Jahren versuchte, durch Verlängerung der Arbeitszeit (1938 häufig 58 bis 65 Stunden pro Woche; Dülffer, 1976, S, 447) und durch eine Intensivierung der Arbeit nicht nur kostengïnstig zu produzieren, sondern hierdurch auch dem Arbeitskräftemangel wirksam zu begegnen. Die Folge war ein ungeheuerlicher Raubbau an der menschlichen Arbeitskraft. Dies ist durch viele Quellen bezeugt. So wird z.B. in einem Bericht über den Bergbau in der Zeitschrift »Der deutsche Volkswirt « auf die »natürlicherweise zunehmende gesundheitliche Anfälligkeit bei hohem Einsatz der Arbeitskräfte« hingewiesen (10.12.1937). Die Überbeanspruchung der Arbeiter führte zu gesundheitlichen Schäden und zu immer häufigeren Arbeitsausfällen.

4. Parallel zum Raubbau der Arbeitskraft ging der Verschleiß an den Maschinen. Die über Jahre voll ausgelasteten Maschinen waren reparaturanfällig geworden (Volkmann, 1976, S.55). Die Maschinen ließen sich bei der übermäßigen Beanspruchung zunehmend nicht mehr in Ordnung halten und wurden »infolge mangelnder Wartung früher [als sonst] betriebsunfähig" (in: Mason, 1975 a, S. 843). ${ }^{2}$ So entstand ein großer Bedarf an Reparaturen und Erneuerungen bei Maschinen (Thomas, 1966, S. 140). Um den ständig steigenden Produktionsausstoß bewerkstelligen zu können, wurden zunehmend ältere Maschinen verwendet. ${ }^{3}$ Deshalb war sogar zu befürchten, daß die Leistungsfähigkeit der Betriebe sinken. 
würde. Eine solche Entwicklung machte sich bereits im Jahre 1938 in verschiedenen Branchen bemerkbar. Vor allem wurde für den Montanbereich ein »anhaltender Rückgang des Arbeitsertrages je Mann je Schicht« verzeichnet (Dt. Volkswirt, 10.12.1937, 20.4.1938, 6.5.1938, 18.3.1938; Mason, 1975 a, S. 596, 572; Volkmann, 1976, S. 55). Auch bei den Hütten und Stahlwerken würden - so hieß es 1938 - sich die Stückkosten erhöhen, die optimale Ausnutzung der Leistungsfähigkeit der Hochöfen sei erreicht bzw. schon überschritten (Dt. Volkswirt, 18.3.1938, 2.9.1938). Ein konkretes Beispiel mag die Entwicklung verdeutlichen: Im Bochumer Verein veränderte sich die Lage beträchtlich. Während man im Jahre 1927 noch einen Umsatz von rund 1200 RM pro beschäftigtem Arbeiter erreicht hatte, waren es im Jahre 1938/39 nurmehr 965 RM, während gleichzeitig der Umsatz je Tonne Rohstahlerzeugung von $116 \mathrm{RM}$ im Jahre 1926/27 auf $184 \mathrm{RM}$ im Jahre 1938/39 angestiegen war. Diese Senkung der Arbeitsproduktivität wurde vor allem durch die Knappheit an gelernten Arbeitskräften bedingt, weshalb das Unternehmen gezwungen wurde, branchenfremde, weniger leistungsfähige und geringer qualifizierte Kräfte einzustellen (Seebold, 1981, S. 96).

5. Ein weiteres wichtiges Krisenphänomen stellte die Disproportionalität der Wirtschaftssektoren dar. Damit ist die völlig ungleiche Investitions- und Produktionssteigerung bei der Produktionsgüterindustrie einerseits und der Konsumgüterindustrie andererseits gemeint. Diese Disproportionalität ging weit über das Maß hinaus, das für konjunkturelle Aurischwungsphasen als »normal « gilt. Zwischen 1935 und 1939 wurden die Investitionen in den Produktionsgüterindustrien verdreifacht und nach dem Herbst 1936 fiel infolge gezielter staatlicher Investitionslenkung (z.B. durch Investitionsverbote in der Konsumgüterindustrie) etwa die Hälfte aller industriellen Anlageinvestitionen in den Bereich der durch den Vierjahresplan kontrollierten rüstungswichtigen Wirtschaftszweige. Zwar erhöhte sich zugleich auch das Mißverhältnis zwischen den beiden volkswirtschaftlichen Sektoren. Deı Anteil der Konsumgüterindustrie am Bruttosozialprodulkt sank von $72 \%$ im Jahre 1935 auf 54\% im Jahre 1939 (Carroll, 1968, S. 184ff.; Erbe, 1958, S. 48ff.).

Eine solche iiberdimensional starke Verschiebung der Produktionsstruktur barg eine immense ökonomische Krisenproblematik für die nächste Zukunft in sich. Die relative Unterentwickeltheit der Konsumgüterindustrie hatte zur Folge, daß die angewachsenen Konsumbedürfnisse sich nicht mehr ausreichend befriedigen ließen. Zwar waren die Einkommenssteigerungen für die breiten Volksschichten in den dreißiger Jahren nicht gerade üppig, doch lag die Kaufkraft im Jahre 1939 um 2 Mrd. RM über dem Vorkrisenniveau von 1929. Dabei ist allerdings auch zu berücksichtigen, daß sich im Zeitraum dieser $10 \mathrm{Jahre}$ die Anzahl der Bevölkerung um 5,5 Mio. Menschen erhöht hat (Mason, 1975 b, S. 170).

Die erhöhte Kaufkraft sollte eigentlich durch Steuererhöhungen oder durch die Zeichnung von Reichsanleihen abgeschöpft werden. Da die Zeichnung der Reichsanleihen den vorgesehenen Zweck nicht voll erfüllte, wären Steuererhöhungen "fällig" gewesen. Doch einen solchen unpopulären Schritt wollte das NS-Regime nicht gehen. So blieben die Steuern auf ihrem relativ niedrigen Stand. Eine Ausnahme stellte lediglich die schrittweise Erhöhung der Körperschaftssteuer von 14 auf 40\% dar (ebd., S. 161; vgl. Boelcke, 1977). Durch die nur begrenzt vorgenommene Abschöpfung wuchs die Konsumnachfrage der Bevöllserung. Unerwünschte Preiserhöhungen konnten trotz Höchstpreisfestsetzungen nicht verhindert werden.

Während in den ersten Jahren nach 1933 die Tatsache, daß die Früchte des Konjunlkturaufschwungs nur in geringem Maße der Bevölkerung zugute kamen, weithin den Menschen 
nicht bewußt war (Sternberg, 1951, S. 240), wuchs mit der Steigerung der Konsumkraft die Kritik, insofern als die Menschen feststellten, daß sie für ihr mühsam verdientes Geld nur minderwertige Ware kaufen konnten. In dieser relativen Mangelsituation entstand auch folgender $»$ Flüsterwitz«:

"Womit soll sich das deutsche Volk im Zeichen des Vierjahresplanes kleiden, wenn auch das Holz für wichtigere Dinge gebraucht wird? Sehr einfach, mit Stoffen, hergestellt aus Hitlers Hirngespinsten, Göbbels Lügengeweben und dem Geduldsfaden des deutschen Volkes« (Gamm, 1980, S., 22).

Damals versuchte das NS-Regime auch, immer genauer die Stimmungslage der Bevölkerung auszukundschaften. Da es keine freie politische Meinungs- und Willensbildung gab, war es gezwungen, auf anderen Wegen zu erfahren, wie das Volk dachte. Über die "Stimmung und Haltung" der deutschen Bevölkerung berichteten regelmäßig die geheime Staatspolizei (Gestapo), der Sicherheitsdienst (SD), die Parteiführungsstellen, die Oberlandesgerichtspräsidenten und Generalstaatsanwälte, die Reichspropagandaämter und das Oberkommando der Wehrmacht. Sie arbeiteten nicht mit Befragungen, sondern mit einem Heer von Spitzeln und Informanten. Allein der Sicherheitsdienst verfügte im Jahre 1937 über etwa 3000 hauptamtliche Mitarbeiter und 30- bis 50000 nebenamtliche anonyme Spitzel. Das bedeutete, daß auf etwa je 2500 Bürger ein SD-Mann kam (Wette, 1979, S. 139). Es ist auffällig, daß gerade im Jahre 1939 dieses Stimmungsberichtssystem weiter »verbessert « worden ist - ein Zeichen für die zunehmende Unsicherheit faschistischer Herrschaftsausübung.

Kennzeichnend für die faschistische Gesamtpolitk in jener Zeit vor und nach Kriegausbruch war die Tatsache, daß die zunehmende Repression und die Systematisierung des Terrors von einer punktuell relativ großen Nachgiebigkeit begleitet wurden (Mason, 1982, S.24). Man wagte nicht recht, die Bevölkerung noch mehr zu belasten, als dies tatsächlich schon der Fall war (ders., 1975 b, S. 176). So ist z.B. überliefert, daß Hitler sich sehr beunruhigt zeigte, als Ende August 1939 Bezugsscheine im Zuge der Lebensmittelrationierung eingeführt wurden, weil er die als wahrscheinlich geltenden negativen Auswirkungen auf die Stimmung der Bevölkerung fürchtete (in: ebd., S. 172; Herbst, 1982, S. 72). So scheute man sich auch, eine allgemeine Frauendienstverpflichtung einzuführen, und gewährte sogar jenen Frauen, deren Männer eingezogen worden waren, relativ hohe finanzielle Unter. stützung. Selbst die Kriegswirtschaftsverordnung vom 4. September 1939, die sich auf die Lohntüte der Arbeiter negativ auswirkte, mußte nach wenigen Monaten z.T. wieder rïckgänn. gig gemacht werden - und dies, obwohl der Krieg bereits ausgebrochen war - weil zu negative Folgen auf die Stimmung der Arbeiter befürchtet wurden (dazu: Mason, 1975 b, S.179).

6. Die Arbeiter reagierten auf die ihre Kräfte immer mehr verzehrende Alltagssituation am Ende der dreißiger Jahre verstärkt mit individualisierten, getarnten Streiks und füllten damit die ihnen noch durch das Terrorregime verbliebenen Aktionsräume aus. (Streng verbotene offene Streiks durchzuführen überforderte wegen der Gewaltandrohung durch die Gestapo die meisten Arbeiter). Der Klassenkampf nahm rudimentäre und verdeckte Formen an. Man bummelte, man führte die zugewiesenen Arbeiten schlecht aus, man war unpünktlich, machte blau, feierte krank (ebd., S.174). Die Alkten sind voll von diesbezüglichen Klagen der Unternehmer und der Bürolsratie. Doch Abhilfe zu schaffen schien schier unmöglich zu sein. Selbst die Gestapo stand solchen verbreiteten stillen Obstruktionsgebärden der Arbeiterschaft relativ hilflos gegenüber. Sie versuchte, durch exemplarischen und 
präventiven Terror (ders., 1982) die Arbeiter im Zaum zu halten. Wahrscheinlich verhinderte sie auf diese Art offene Streiks, aber die individuell getarnten Streiks konnte sie dadurch nicht aus der Welt schaffen und materielle Anreize auf breiter Basis kamen aus aufrüstungspolitischen Interessen ebenfalls nicht infrage. Die Lohnquote, die man so kunstvoll seit Bestehen des Systems trotz aufsteigender Konjunktur heruntergedrüclkt hatte, sollte sich ja nicht erhöhen, wenn überhaupt, dann eher noch verringern.

7. So häuften sich gegen Ende der dreißiger Jahre auf ökonomischem und sozialem Gebiet die Probleme und schankelten sich gegenseitig hoch. Sie wurden auch bereits auf politischer Ebene sichtbar. Die Tatsache z.B., daß immer wieder neue Ämter wie Pilze aus dem Boden schossen, (z.B. die Vierjahresplanbehörden) und daß immer wieder neue Sonderbevollmächtigte ernannt wurden, deutet auf zunehmende Steuerungsprobleme des Herrschaftssystems hin. Polykratie wird dieses Kompetenzchaos in der Literatur genannt, und diese entstand letztlich, weil die gesellschaftlichen und die gesellschaftspolitischen Widersprüche sowie die überhöhten Anforderungen an Mensch und Maschine immer schwerer zu steuern waren. Zwar vermochte das NS-Regime dadurch eine beachtliche Flexibilität und Effizienz in Teilbereichen, z.B. bezuiglich des Baus des Westwalls, an den Tag zu legen. Aber die negativen Steuerungsfolgen sind ebenfalls unübersehbar (Herbst, 1978, S.390). 8. Bleibt die Frage offen, wie die Situation im Jahre 1938/39 insgesamt zu beurteilen ist. Darüber gibt es in der Literatur unterschiedliche Auffassungen. Herbst vertritt die Position, daß es sich um Engpässe und Teillkrisen, bedingt durch die Schwächen des Steuerungssystems gehandelt habe:

»Die bisher vorliegenden Forschungsergebnisse lassen den sicheren Schluß, daß sich die innere Lage des nationalsozialistischen Deutschlands am Vorabend des Krieges soweit zugespitzt hatte, daß man von einer allgemeinen Krise des Systems sprechen kann, nicht zu« (ebd., S.388).

Während Herbst nur Einzelkrisen zu erkennen glaubt, mehren sich die Stimmen, die die damalige Situation durch eine Gesamtkrise gekennzeichnet sehen wollen. An erster Stelle ist T. Mason zu nennen. Er kam 1975 aufgrund umfangreicher Untersuchungen zu dem Schluß:

„Sehr viel länger als bis zum Herbst 1939 hätte das Dritte Reich nicht abwarten können, um aus der inneren Krisenlage auszubrechen - bis 1943 auf gar keinen Fall. Ohne diese Flucht nach vorn gab es nicht einmal die Möglichkeit, im Innern die Repressionen so zu steigern, wie es die Fortführung der Aufrüstung und die Vermeidung wirtschaftlicher Krisen erforderten« (1975, S. 182).

Dülffer kennzeichnet die Situation ähnlich:

"Ein Grenzwert, der ein Umkippen in soziale Unruhen größeren Ausmaßes oder gar in eine revolutionäre Situation wahrscheinlich gemacht hätte, war unmittelbar nicht gegeben. Auf längere Sicht, d.h. noch über. mehrere Jahre hinweg, war freilich die Diskrepanz zwischen dem Umfang aller öffentlichen Aufträge, zumal der Rüstung, und den Leistungsmöglichleiten der Wirtschaft unüberbrückbar (...)«(1976, S. 469).

Auch Dülffer vertritt die Auffassung, daß im Jahre 1939 Grenzwerte erreicht worden seinen (ebd., S. 451):

„Es waren also die innere Krise und die durch Wettrüsten gekennzeichnete internationale Situation, die 1939 zum Kriege trieben (...)« (ebd., S. 469; ähnlich Petzina, 1975, S. 80).

Dülffer kommt also - ähnlich wie Mason - zu dem Schluß, daß die NS-Machtelite aus bei- 
den Krisenlagen heraus mit der Entfesselung eines Raubkrieges »die Flucht nach vorn« angetreten habe (Dülffer, 1976. S.470).

Das Erreichen von Grenzwerten betont auch Blaich:

„Langfristig freilich wäre der Weg der Rüstungsfinanzierung durch das Versprechen einer zukünftigen besseren Versorgung mit Zivilgütern natürlich nicht gangbar gewesen« (1979, S. 59; ähnlich Peukert, 1982, S. 82; Deist, 1979, S.444; Geyer, 1980, S. 448).

Schließlich sei noch auf die Sammelbände hingewiesen, die das militärgeschichtliche Forschungsamt in Freiburg zu diesem Thema herausgegeben hat. (Forstmeier/Volkmann, 1975; dies., 1977; Deist u.a., 1979). Von besonderer Bedeutung für die hier aufgeworfene Fragestellung nach dem Krisencharakter der unmittelbaren Vorkriegszeit sind die Überlegungen von Hans-Erich Volkmann. Auch er kommt zu dem Urteil, daß die volkswirtschaftlichen Reserven damals weitgehend erschöpft waren und eine »kritische gesamtöko" nomische Konstellation « (1976, S.56) eingetreten war.

Nimmt man ferner die kritischen Wahrnehmungen und deren marx-orientierte Verarbeitung durch Sohn-Rethel (1973) und - in seiner Nachfolge stehend - die Überlegungen von Kadritzke (1976) hinzu, so spricht vieles dafür, daß sich dieses Analyseergebnis mit guten Argumenten - wenn auch mit unterschiedlicher theoretischer Verankerung - mehr und mehr in der Geschichtswissenschaft durchsetzen wird.

\section{Krisenpermanenz}

Im frigenden Teil soll auf einige Konstitutionsfaktoren, die für die Beurteilung der Gesamtkrise von 1938/39 wichtig sind, in einer Art Längsschnitt eingegangen werden. Damit im Zusammenhang steht die Frage, ob von einer Krisenpermanenz des NS-Herrschaftssystems gesprochen werden kann.

\section{1. Ökonomie und Politik}

Hinsichtlich der wirtschaftlichen Entwicklung ist ein Rückblick auf die Geburtsstunde, genauer auf die Geburtsfehler des NS-Systems notwendig. So ist zu fragen, ob nicht schon die Art und Weise, in der damals - 1933/34 - die große Wirtschaftskrise von den Nationalsozialisten angegangen worden ist, die Krise am Ende der dreißiger Jahre verursacht hat. Mittlerweile besteht Einigkeit in der Literatur darüber, daß - trotz bestimmer ziviler Arbeitsbeschaffungsmaßnahmen zu Beginn des »Dritten Reiches« - die forcierte Aufriistung von Anfang an im Mittelpunkt der faschistischen Wirtschaftspolitik gelegen hat (so schon W. Fischer, 1968, S.61). Die forcierte Aufrüstung befriedigte sowohl die expansionsorientierten als auch die betriebsökonomisch ausgerichteten Interessen. Letztere resultierten aus dem Charakter der Großen Krise, die seit 1929 immer verheerender um sich gegriffen hatte. Diese Krise erhielt vor allem durch die im Zuge der Hochkonjunktur von 1924/25 bis 1929 vorgenommenen großen Investitionen und den damit einhergehenden Rationalisierungen eine besondere Tiefen- und Breitenwirkung, insofern sie bei einer sich seit 1929 rasant verschlechternden Weltmarktentwicklung große Überkapazitäten schufen.

Solche Rationalisierungen und Betriebsvergrößerungen ließen die fixen Kosten beträcht- 
lich steigen. Diese stark erhöhten fixen Kosten blieben auch dann erhalten, als in der großen Wirtschaftskrise der erhöhten Produktionskapazität die entsprechenden Absatzchancen fehlten. Ein solches Mißverhältnis regulierte sich nicht mehr durch die Gesetze der freien Marktwirtschaft selbst, sondern bedurfe staatlicher Eingriffe (Sohn-Rethel, 1973; Volkmann, 1978, S.278ff.). Für die konjunkturelle Wiederbelebung einer Wirtschaft, die im wahrsten Sinne des Wortes - trotz leichten konjunkturellen Anstiegs seit dem Sommer 1932 - noch immer auf dem Boden lag, war deshalb die massive Rüstungspolitik der entscheidende Krisenlösungsfaktor ${ }^{5}$, der im Interesse des wichtigsten Teils der Kapitalbesitzer lag. Dieses aktuelle Interesse an der Rüstungskonjunktur wurde noch durch weitergehende Interessengemeinsamkeiten mit der NS-Parteiführung unterstützt:

»Aus der Sicht der Unternehmer galten allein zwei Tatsachen als Kriterien für eine positive Bewertung der »Wehrwirtschaft«, nämlich einmal der Verbleib der Produlstionsmittel in ihrem Eigentum, verbunden mit der Möglichkeit der Gewinnmaximierung und zum anderen das Versprechen der Nationalsozialisten, die aus der Weimarer Republik übernommenen ökonomischen Probleme zu lösen, und zwar durch territoriale Expansion und Markterweiterung« (ebd., S.289).

Die forcierte Rüstungskonjunktur verursachte - vor allem mit dem Vierjahresplan seit 1936 - jene Krisenphänomene, von denen schon die Rede war. Hinzu kamen Exportmüdigkeit und Exportverdrossenheit der deutschen Industrie (s.u.), eine zwar nicht gewollte, aber in Anbetracht der nicht sehr guten Weltmarktsituation kaum vermeidbare Folge der einseitigen Binnenmarktorientiertheit des neuen Konjunkturaufschwungs. Insgesamt gesehen läßt sich also feststellen, daß jene Engpässe und Widersprüche, die sich am Ende der dreißiger Jahre zu einer Gesamtkrise verdichtet haben, von Anfang an in der faschistischen Wirtschaftspolitik angelegt waren, und zwar durch die Art und Weise, wie auf die große Wirtschaftskrise von 1929 ff. reagiert worden ist. Das heißt, die ökonomische Krise von 1929ff, wurde nicht wirklich gelöst, sondern nur verlagert und vertagt (Kadritzke, 1976, S.168). Zwar konnten die Arbeitslosigkeit und das Brachliegen von Industriekapazitäten die auffälligsten Krisenerscheinungen der sogenannten Großen Krise von 1929ff. - sehr wohl beseitigt werden; gleichzeitig traten jedoch neue Krisenphänomene in anderen Bereichen hervor.6 Wie ein Maulwurf bohrte sich die Krise im Boden der Volkswirtschaft weiter, einmal an dieser Ecke an die Oberfläche kommend, das andere Mal an jener. Der Faschismus hat, so kann man zusammenfassend sagen, die "Disproportionalitäten der Ausgangslage hemmungslos vervielfacht" (Sohn-Rethel). T. Mason spricht in diesem Zusammenhang von einer »Kettenwirkung der Mangelerscheinungen«, von einer Art »negativem Multiplilkatoreffekt« (1975 (b), S.171).

Mit jedem Jahr, in dem die deutsche Wirtschaft sich mehr und intensiver auf die Kriegsproduktion einstellte, wurde es schwieriger, auf diesem Wege anzuhalten, geschweige denn Alternativwege zu beschreiten. Es ist nicht auszuschließen, daß es so etwas wie einen ökonomischen "point of no return « gegeben hat (s.u.). Selbst Herbst spricht von einer Einbahnstraße: Je weiter man auf dieser Einbahnstraße fortschritt, »desto geringer wurden die Wahlmöglichkeiten natürlich objektiv« (1982, S.81). Die forcierte Aufrüstungspolitik veränderte die Wirtschaftsstruktur so tiefgreifend und letztlich in so destruktiver Weise, daß eine Umkehr in Anbetracht der schlechten Weltmarktsituation immer schwerer möglich wurde.

Sicherlich gibt es zu einer solchen Betrachtungsweise auch diskussionswürdige Gegenargumente. Nicht die Art und Weise, wie die Große Wirtschaftskrise von 1929ff. überwunden 
wurde, sei maßgeblich Schuld an dem ökonomischen Fiasko der späten dreißiger Jahre gewesen, sondern dieses sei den massiven Fehlentscheidungen des NS-Regimes geschuldet. Als eine solche massive Fehlentscheidung gilt in der Literatur immer wieder die Tatsache, daß Deutschland keine Abwertung der Reichsmark vorgenommen und deshalb die Exportchancen verringert habe (s.u.) sowie die Tatsache, daß durch die Transfermoratorien wichtige Handelspartner, wie die Vereinigten Staaten und Großbritannien, unnötig verärgert worden seien (s.u.; Radkau, 1976, S. 40ff; Schweitzer, 1964; in fragender Form: W. Fischer, 1975, S.133). Als der systemimmanenten Logik der NS-Herrschaft widersprechende Fehlentscheidung wird ferner die halbherzig vorgenommene Lenkungs- und Planungspolitik angesehen. Dazu Herbst:

"Zwar gestalteten sich die rohstoffwirtschaftlichen Voraussetzungen Deutschlands nicht günstig, und die Arbeitskräftereserven schienen eng begrenzt, aber im Rahmen einer streng nach Prioritäten ausgerichteten Lenkung und einer vorausschauenden Planung hätten sich größere Spielräume eröffnen lassen, als praktisch genutzt wurden. Sofern es nur gelang, den Mangel zu bewirtschaften, waren Ausmaß und T'empo der deutschen Aufrüstung vor dem zweiten Weltkrieg nicht primär von der ökonomischen Seite her begrenzt" (1978, S.63; fragend: W. Fischer, 1975, S.134).

Die Analysen stützen sich auf entsprechende Auffassungen, die schon von Zeitgenossen vertreten worden sind (s.u.). Am gewichtigsten ist wohl die von Herbst anvisierte systemimmanente Alternativpolitik. Seine Argumentation läuft nicht nur seiner eigenen "Einbahn-These« zuwider, sondern weist darüber hinaus auch eine voluntaristische Einfärbung auf. Denn bei dieser Auffassung wird die Ökonomie als ein von der Gesellschafts- und Herrschaftsstruktur losgelöster Teilbereich gesehen, anstatt nach den offensichtlich nur begrenzten Handlungsmöglichkeiten und dem inhaltlichen Systemzusammenhang des deutschen Faschismus zu fragen.

\section{Beherrschte und Herrschende}

Kann man also insgesamt gesehen durchaus von einer ökonomischen Krisenpermanenz sprechen, so soll im folgenden Abschnitt skizziert werden, wie sich das Verhältnis von Beherrschten und Herrschenden umreißen läßt. Gefragt wird nach der 'Legitimationsstruktur des deutschen Faschismus.

Deren Wirkungsmöglichkeiten und Wirkungsgrenzen sind freilich schwer einzuschätzen. Insgesamt ist wohl davon auszugehen, daß es den Machthabern zu keinem Zeitpunkt gelungen ist, die Arbeiterschaft in die angebliche Volksgemeinschaft zu integrieren, allenfalls gelang ihnen eine höchst instabile »Bändigung« (Mason, 1982, S. 19). Terror und Gewalt waren $\mathrm{zu}$ allgegenwärtig. $Z$ wang und Lähmung, aber auch Bestechung und Befriedigung (ebd., S. 18) haben es zwar vermocht, daß die Arbeiterschaft sich relativ ruhig verhielt und es zu keinen größeren Streikbewegungen und keiner größeren Zerstörung industrieller Produktionsanlagen gekommen ist (ebd., S.46). Sie haben aber nicht vermocht, die Arbeiterschaft so weit zu instrumentalisieren, daß eine ins uferlose gehende Aufrüstungspolitik in »Friedenszeit « auf Dauer hätte riskiert werden können.

Derweilen hatte sich das Regime durchaus bemüht, die Arbeiter für sich zu gewinnen. Es machte Anstrengungen, die Arbeiter zumindest zu domestizieren. »Voraussetzung« dazu war freilich zum einen die Zerschlagung der autonomen traditionsreichen Arbeiterorgani- 
sationen, zum anderen galt es, auch die arbeiterkulturellen Vereine zu zerstören, ein Eingriff, der sich bemerkenswerterweise nach 1945 als irreversibel erweisen sollte. Terror und Terrorbedrohung sorgten dafür, daß unter den Arbeitern ein hohes Maß an Mißtrauen untereinander entstehen konnte, dem auch viele gewachsene Solidaritätsstrukturen zum Opfer fielen.

Zudem wurde die Fragmentierung der Arbeiterschaft vorangetrieben. In diesem Zusammenhang scheint die damals starke Forcierung des Leistungsgedankens in Form von (konkurrierenden) Individualleistungen von besonderer Bedeutung zu sein. $\mathrm{Zu}$ denken ist dabei an die schon 1933 eingefïhrten Reichsberufswettkämpfe. Die Sieger wurden öffentlich geehrt, beruflich und finanziell gefördert (Schoenbaum, 1968, S.133f.; die Bedeutung herunterspielend: Mason, 1982, S.28). Es wurden neue Leistungsanreize geschaffen, und ab 1939 versuchte das Reichsarbeitsministerium, die sogenannten Lohndifferenzierungsskalen $\mathrm{zu}$ verfeinern, um auch so die Arbeiter verstärkt auf konkurrierende Individualleistungen $\mathrm{zu}$ "trimmen«? In einem Erlaß vom 4.9.1939 forderte das Reichsarbeitsministerium die Reichstreuhänder der Arbeiter hinsichtlich der neuen Tarifordnungen auf:

"Auf eine ausreichende Aufgliederung der Arbeiterschaft und der Lohnsätze ist hierbei Bedacht zu nehmen« (in: Siegel, 1982, S. 95).

1940 schrieb der Reichsarbeitsminister:

"... mehr als bisher muß die Entlohnung in Zukunft von der Wertigkeit der Arbeit abhängig gemacht werden. In neuen Lohngruppen müssen alle Arbeitsvorgänge gleichen Wertes zusammengefaßt werden, um so dem Arbeiter eine bessere Aufstiegsmöglichkeit zu bieten und damit den Anreiz zu weiterer Leistungssteigerung auszulösen. Außerdem lehrt die Erfahrung, daß nur bei weiterer Aufgliederung der Löhne der Lohnstand wirklich gehalten werden kann« (in: ebd., S.95).

Durch eine solche Lohn-Neubemessung sollten zwei Fliegen auf einmal geschlagen werden: Beibehaltung und womöglich Senkung des Lohnvolumens zum einen - Steigerung der Individualleistungen der Arbeiter zum anderen. Gewiß sind diese staatlichen Bestrebungen erst nach Kriegsbeginn zur Wirkung gekommen (darauf verweist Mason, 1982, S.29f.), doch bleibt noch zu untersuchen, inwieweit solche Tendenzen nicht auch schon vor 1939 auf einzelbetrieblicher Ebene zum Zuge gekommen sind.

Offen ist auch noch die Frage, inwieweit diese Lohndifferenzierungspolitik die Fragmentierung der Arbeiterklasse tatsächlich verstärkt hat. Mason (1982) ist hier skeptischer als Siegel (1982). Interessant ist in diesem Zusammenhang die Feststellung des Generalbevollmächtigten für den Arbeitseinsatz, Sauckel, vom September 1942, daß durch die probeweise Überprüfung der Akkorde und die Einführung von acht Lohngruppen in einzelnen Betrieben der Rüstungswirtschaft Leistungssteigerungen erzielt worden seien, »die in der Regel bei $20 \%$, sehr oft jedoch bei wesentlich höheren Prozentsätzen« lagen (in: Siegel, 1982, S.96).

Das Regime versuchte aber auch, den durch Terror herbeigeführten Trümmerhaufen gewachsener Sozialbeziehungen in ihrem Sinne neu zu organisieren. Dazu gehörten kleinere sozialpolitische Verbesserungen. Wenn auch die sich in engen Grenzen haltenden Lohnverbesserungen mehr der Entwicklung der Ware Arbeitskraft zur absoluten Mangelware (nach 1936) geschuldet war, so trugen diese doch wohl schon dazu bei, die zunehmende Arbeitsintensivierung sowie den verlängerten Arbeitstag zu »versüßen« (vgl. Mason, 1975 b, S. 169; Blaich, 1979, S.40). In diesem Zusammenhang ist beispielsweise auch zu erwähnen, daß im Jahre 1937 die Bezahlung der gesetzlichen Feiertage für Lohnarbeiter, also auch die 
Bezahlung des 1. Mai, eingeführt worden ist. - Ferner muß an bestimmte sozialpolitische Angebote und den positiven Wirkungen ihrer propagandistischen Ausschlachtung gedacht werden, wobei die dadurch der öffentlichen Hand bzw. den Unternehmern zufallenden finanziellen Mehrbelastungen auch nicht im entferntesten die Senkung der Lohnquote ausgleichen konnten. Erinnert sei in diesem Zusammenhang an Verbesserungen der betrieblichen Ausstattung, die wohl weniger dem Arbeitsplatz als vielmehr der Gestaltung der Nicht-Arbeitszeit dienten (bessere Kantinen und Waschräume, Werkssportplätze, Schwimmbäder u.a.m.). Dahinter stand der Versuch, die zunehmende Arbeitsintensivierung durch verbesserte Freizeitangebote erträglicher zu machen (dazu neuerdings Friemert, 1980) und die Arbeiter so in das System besser einzubinden. Überdies trug ein Teil der betrieblichen Sozialverbesserungen zur »sekundären Ideologisierung « der Arbeiter bei (ebd., S.250ff.).

Wenn beispielsweise ein Betriebs-Schwimmbad von der Belegschaft in nichtbezahlter Gemeinschaftsarbeit gebaut und anschließend benutzt wurde, gerieten leicht jene in eine unerwünschte Außenseiterposition, die aus vielleicht politischen Gründen weder an der Gemeinschaftsarbeit noch an der gemeinschaftlichen Benutzung teilnahmen. Vermutlich hielten viele Arbeitskollegen solchem Druck seitens der Kollegen-Gemeinschaft auf einem ja nach außen hin völlig unpolitisch erscheinenden Gebiet nicht stand; so gelang es wohl nicht selten, diese »ohne offene äußere Gewalt, sozusagen unter sich, in die Nazi-Form zu zwingen « (ebda., S.253).

Auf außerbetrieblicher Ebene stellte das Kraft-durch-Freude (KdF)-Freizeitprogramm das auch für Arbeiter attraktivste Angebot faschistischer Sozialpolitik dar. Vor allem gilt dies für die auch unter Arbeitern beliebten KdF-Reisen. Natürlich konnten sich die wenigsten Arbeiter tatsächlich solche KdF-Reisen leisten, vor allem nicht die gerade propagandistisch so sehr ausgeschlachteten größeren (Schiffs-)Reisen (Spode, 1982), noch weniger konnten die Arbeiter an den Kauf eines Volkswagen denken. Doch war mit diesen Angeboten etwas Neuartiges geschehen. Die Dinge rückten nun überhaupt für den Arbeiter in erreichbare Nähe, und so konnten viele, die damals noch keine größere KdF-Reise bezahlen konnten, die Hoffnung haben, daß dies nurmehr eine Frage der Zeit sein würde (vgl. Herbst, 1978, S.383). Wie ïberhaupt die Zeit des »Dritten Reiches« durch eine starke ökonomische, soziale und politische Dynamik ausgezeichnet schien, woraus die verbreitete Hoffnung der Menschen auf eine weitere Verbesserung ihrer Alltagssituation resultierte. Von einer solchen Dynamik gingen (wenn auch zeitlich begrenzt $\hat{e}^{\text {) }}$ systemstabilisierende Wirkungen aus, ungeachtet der Tatsache, daß die Dynamik selbst als Ausdruck der permanenten inneren Krise des faschistischen Systems aufzufassen ist.

Diese Hoffnung auf eine Aufwärtsentwicklung war ebenso bei den Mittelschichten vorhanden. Die kolossale ökonomische und soziale Dynamik, die das »Dritte Reich « auszeichnete, förderte ein häufig zu beobachtendes systemloyales Verhalten vieler Angestellter und Beamter (soweit sie nicht geschwerkschaftlich organisiert waren), zumal wenn damit Aufstiegs" und Karrierehoffnungen verbunden waren (einführend: Peukert, 1982, S.110ff.). Auch wenn die mittelstandspolitischen Maximalforderungen nach 1933 politisch nicht zum Zuge kamen, auch wenn der Aufwärtstrend unerwartet langsam vor sich ging, so waren doch wichtige, bisher vergeblich gestellte mittelstandspolitische Forderungen für Handwerk und Einzelhandel zumindest teilweise - nicht zuletzt auf Kosten der Kleinstbetriebe - durchgesetzt worden (dazu und zum folgenden vgl. v. Saldern, 1979). Die größeren und leistungsfähigen Kleinbetriebe konnten überdies - wenn auch höchst dispropor- 
tional zur Großindustrie - von der Rüstungskonjunktur (und teilweise sogar von der sog. landwirtschaftlichen Erzeugungsschlacht) "profitieren «. Die »soziale Funktion« des Faschismus bestand eben nicht nur in der Bedürfnisbefriedigung der Großindustrie, sondern darüber hinaus auch in einer rüstungs- und expansionsorientierten Krisen»lösung " für die Gesamtwirtschaft, wozu ein bestimmter Teil der Kleinproduktion gehörte. Insofern muß die These vom Widerspruch zwischen »sozialer Funktion« und »sozialer Basis« des Faschismus (z.B. Kühnl) neu überdacht werden. (Eindeutig negativ verlief die Entwicklung »nur« im Handwerk, Einzelhandel und in der Landwirtschaft für die (vielen) Kleinstbetriebsinhaber. Diese führten entweder weiterhin eine wirtschaftliche Kümmerexistenz oder mußten ihren Betrieb ganz aufgeben (Umlegungsaktionen und Flurbereinigungen in der Landwirtschaft, Schließungsaktionen im Einzelhandel und Handwerk).) Die zwar von oben nach dem Führerprinzip gelenkten, aber »von unten« im großen und ganzen durchaus mitgetragenen Berufsstandorganisationen - die Kammern sowie der Reichsnährstand - sorgten für eine relative Stabilisierung des Regimes in diesen Bereichen. Die soziale Aufwertung des Handwerksberufs und der bäuerlichen Arbeit sowie der Versuch, die soziale Rangordnung von der volkswirtschaftlichen Potenz abzukoppeln, war ebenfalls wohl nicht ohne systemstabilisierende Wirkungen geblieben. Trotz aller (berechtigter) »Meckerei« und Kritik an bestimmten Verordnungen und Entwicklungen gehörte der »alte Mittelstand (worunter der lebens- und leistungsfähige Kleinbetriebsinhaber gemeint ist) auch nach $1933 \mathrm{zu}$ den zwar häufig nicht parteigebundenen, aber im Grunde systemloyalen Stützen des Faschismus. Im politischen Widerstand fehlte er fast gänzlich.

Ein solches Sich-Einrichten im faschistischen Deutschland schloß jedoch nicht das Umsichgreifen beträchtlicher Widerspruchswahrnehmungen zwischen Schein und Realität sowie die (innere) Ablehnung bestimmter politischer Maßnahmen (Repression gegen die Kirche, Nürnberger Gesetze, Kristallnacht u.a.m.) aus. Doch viele Menschen konnten (und wollten) ihre situativen Widerspruchswahrnehmungen und Teilkritiken nicht in einen politischen Gesamtzusammenhang bringen, wobei freilich beachtet werden muß, daß die Informations- und Aufklärungsmöglichkeiten damals äußerst erschwert waren.

Überdies darf nicht vergessen werden, daß das NS-Regime stets beflissen war, die Schwachund Bruchstellen des Systems bestmöglich zu verschleiern. - Beispiele für solche Legitimationsbestrebungen sind die sorgsame Währungsimagepflege, die auffällige Betonung der Friedensliebe der deutschen Regierung, ferner das Herunterspielen der Devisenkrise von 1935 in der Öffentlichkeit. (Göring entschied damals bekanntlich, daß die vorhandenen äußerst knappen Devisen nicht für die Beschaffung der dringend benötigten Rohstoffe zur Sicherung der Aufrüstungsproduktion, sondern vorübergehend für die Beschaffung unumgänglich wichtiger Lebensmittel ausgegeben werden sollten (Petzina, 1968, S.33).)

Dreh- und Angelpunkt aller Legitimationsbestrebungen des Faschismus war jedoch die charismatisch wirkende Führergestalt (dazu: Kershaw, 1980). Ihr kam die Funktion zu,' der »ruhende Pol« in einem System zu sein, dessen Chance zur Legitimierung allein in der dynamisch-offensiven Bewegung (und letztlich im siegreichen Kampf) gelegen hat. Das Führer-Charisma wurde vor allem durch die außenpolitischen Erfolge gestärkt. Zu dessen ständiger Reproduktion waren Propaganda und Massenaufzüge notwendig. Untermauert wurde die Führerposition vor allem durch ideologische Praxis. Hier sind die vielen Versuche zu nennen, die darauf hinausliefen, im Ritual die Klassengesellschaft durch die Volksgemeinschaft zu ersetzen - in der Hoffnung, die Menschen würden im VolksgemeinschaftsRitual einen vollwertigen Ersatz für die real fortexistierende Klassengesellschaft sehen. Be- 
sondere Bedeutung kommt in diesem Zusammenhang dem Opferritual zu (z.B. Winterhilfswerk; dazu: Elfferding, 1982, S.199ff.). So gelang es dem Faschismus, »eine Legitimationsstruktur aufzubauen, die eben nicht nur vom Terror abhängig war. Allerdings: Was in der Organisierung der Masse nicht lösbar war, wurde durch ihre unmittelbare Unterdrückung 'erledigt'« (Blanke, 1977, S.136f.).

Wie stark die Wirkung war, die von solchen ideologischen Praxen ausging, läßt sich schwer abschätzen. Jedenfalls gab es noch immer eine große Anzahl von Menschen, die weder dadurch noch durch andere Legitimationsversuche des Regimes ihre Überzeugung aufgaben, daß das NS-System ein Unrechtssystem sei, und die sich deshalb innerlich auch nicht »einfangen « ließen, sondern - so gut es ging - eine nichtfaschistische bzw. antifaschistische Gegenkultur aufrechterhielten. Hunderttausende von Menschen - vor allem aus der sozialist ischen und kommunistischen Arbeiterbewegung - wurden aus politischen Gründen verfolgt.

So eindrucksvoll die absoluten Zahlen auch sind, so darf aber nicht vergessen werden, daß der Schritt in den aktiven politischen Widerstand "nur« von Minderheiten getan wurde. Eine ernsthafte Bedrohung für das Regime ging von den Widerstandsgruppen nicht aus. Die Gest apo saß am längeren Hebel. Durch Terror und Terrorbedrohung gelang es ihr, die Rückkoppelungsmöglichkeiten zwischen den politischen Widerstandsgruppen und größeren Bevölkerungsteilen gewaltsam auf ein Minimum zu reduzieren und auch die Aktionsräume zur Aufrechterhaltung und Stabilisierung antifaschistischer. Gegenkultur immer mehr zu beschneiden. (Hier ist z.B. auf die Eingriffe in das katholische Sozialmilieu seit 1937 hinzuweisen.) Durch ein gut funktionierendes Überwachungs- und Bespitzelungssystem war der politische Widerstand auf die Arbeit in Kleinstgruppen beschränkt. Der Zwang zur Tarnung und zur Anonymität verminderte die Erfolgschancen (dazu: Löwenthal/v.d. Mülllen, 1982; Kleßmann/Pingel, 1980). Von der Organisierung eines allgemeinen Volksaufstandes war man weit entfernt. Umso größeres Gewicht bekam das Verhalten der Herrschaftseliten; doch hier war in den ersten Jahren des Regimes nichts von politischem Widerstand zu merken, und die populistischen Strömungen in der NSDAP waren seit dem Schlag gegen Röhm und die SA im Jahre 1934 entscheidend geschwächt worden. Doch der Grad der dadurch erreichten Systemstabilisierung ist trotzdem sehr gering geblieben. Denn die Interessendifferenzen der kapitalistischen Industriegesellschaft wurden dan mit nicht aufgehoben, sondern in die Teileliten, vor allem auch in die "Einheits«-Partei selbst, verlagert (dazu: Blanke, 1977, S. 135f.). Deshalb arbeiteten die konkurrierenden Teileliten in Wirtschaft, Militär, Bürokratie und Partei im Herrschaftsalltag oftmals mehr gegen- als miteinander. Die Parteiführung versuchte, dem Auseinanderdriften der Interessen durch sog. "Nazifizierung « der von den traditionellen Teileliten beherrschten Bereiche entgegenzusteuern, was diese zu verhindern versuchten, bzw. womit sich diese nur ungern abfanden. Die Parteispitze versuchte ferner, die potentielle Systemgefährdung, die durch die konkurrierenden Teilinteressen entstand (gegenseitige Blockierungsgefahr), durch die Schaffung neuer, von der NS-Führung besetzter Ämter zu konterkarieren (System der Sonderbevollmächtigten). Solche polykratischen Grundzüge des Herrschaftssystems förderten zwar eine gewisse Flexibilität des Regimes, vergrößerten aber auch dessen Labilität.

Die potentielle Gefährlichkeit der konkurrierenden Teileliten wurde jedoch durch die funktionelle Abhängigkeit vieler »Wirtschaftsführer« von den staatlich vergebenen Rüstungsaufträgen und der dadurch bedingten inneren und äußeren Abhängigkeit vom politischen System sowie der einzelbetriebszentrierten Interessenverfolgung politisch weitge- 
hend paralysiert. Es ist wohl deshalb kein Zufall, daß aus diesen Kreisen kein nennenswerter Beitrag zum politischen Widerstand gegen das faschistische Deutschland geleistet wurde. - Auch die deutsche Militärtradition bot

„im Grunde kein tragfähiges Fundament für einen politischen Widerstand.« Dieser blieb deshalb auf die vor dem Krieg isolierte »Einzelinitiative geistig und moralisch selbständiger Militärs beschränkt« (Bracher, 1982, S. 147).

Die zunehmende Verstrickung vieler Herrschaftsträger in Unrechtshandlungen und Verbrechen des Systems verringerten die Chance, sich aus rechtsstaatlichen und humanitären Motiven von diesem Herrschaftssystem abzuwenden.

Zusammenfassend läßt sich feststellen, daß der politische Widerstand dem NS-System in den Jahren vor dem Krieg nicht wirklich gefährlich wurde. Seine Bedeutung liegt deshalb zum einen darin, daß es ihn überhaupt gab. Zum anderen zwang er das Regime zur terroristischen Form der Herrschaftsstabilisierung, womit er dessen Legitimationskraft wesentlich schwächte und dessen Instabilisierung förderte. Auch von daher gesehen kann man von einer Krisenpermanenz des deutschen Faschismus sprechen - trotz der noch immer beachtlichen (nicht-terroristischen) Legitimationserfolge.

\section{Krisenbewußtsein und Krisenlösungsvorschläge}

Während bisher die Strukturen und Prozesse aus der Retrospektive analysiert wurden, soll nun skizziert werden, $o b$ und ggf. wie die Bevölkerung bzw. die Herrschaftseliten die Gesamtkrise um 1938/39 wahrnahmen und welche Krisenlösungsvorschläge damals gemacht wurden.

Nach all dem, was wir über diese Phase vor Kriegsbeginn wissen, hat die Bevölkerung am Ende der dreißiger Jahre im allgemeinen kein Bewußtsein davon gehabt, daß sich das System in einer Gesamtkrise befinden würde. Zwar erfuhren viele häufig am eigenen Leib die Teilkrisen und die Engpässe vor allem in der Versorgung und am Arbeitsmarkt, doch die mangelnden Informations- und Aufklärungsmöglichkeiten machten es für den Mann und die Frau auf der Straße kaum möglich, hierin den Ausdruck einer Gesamtkrise zu erkennen. Von daher gerieten die Herrschaftsträger nicht in Zugzwang, doch die Frage stellte sich für sie, wie lange der kunstvoll über die Gesamtkrise verbreitete Schleier noch seine verhüllende Funktion erfüllen würde (vgl. Peukert, 1982, S. 82). Hinzu kam die damals schon weit verbreitete Furcht der Bevölkerung vor einem in naher Zukunft ausbrechenden Krieg, auch wenn man dabei vor allem die sich verschärfende außenpolitische Gesamtkonstellation und nicht die sozio-ökonomischen Zwangslagen im Innern des Landes im Blick hatte. Doch da ein Krieg unpopulär war, war auch die Furcht der Bevölkerung vor dem Krieg für die Machthaber nicht ganz ungefährlich.

Bedeutend krisenbewußter als die Bevölkerung es sein konnte, waren diejenigen, die in der Lage waren, die wirtschaftliche Situation zu beurteilen und eine wirtschaftliche Zukunftsprognose aufzustellen (Mason, 1975 a, S.216). Die Krisenanalysen und Krisenlösungsvorschläge aus damaliger Zeit lassen drei unterschiedliche Richtungen erkennen:

1. Von verschiedener Seite wurde vor allem an der so stark forcierten Aufrüstungspolitik sowie an der deutschen Außenhandelspolitik Kritik geubt. In diesem Zusammenhang sind der Reichswirtschaftsminister und Reichsbankpräsident Schacht, der Reichsarbeitsminister 
Seldte, der Reichssparkommissar Goerdeler, der Reichsfinanzminister Schwerin von Krosigk sowie der Generalstabschef Beck zu nennen. Sie verlangten, daß Ausmaß und Tempo der Rüstungsproduktion gedämpft bzw. verlangsamt und so den Größenordnungen der vorhandenen Wirtschaftsreserven angepaßt werden mögen. Überdies seien die Exportbemühungen zu verstärken. Geheimrat Bücher, Direktor eines großen Elektrokonzerns, verfaßte in den Jahren 1934 und 1935 z.B. zwei Memoranden, in denen er für eine Abwertung und für eine enge Kooperation mit den anderen Welthandelspartnern plädierte (Doering, 1959, S.223). Hier kam demnach ein Vertreter jener Gruppe zu Wort, die sich auf dem Weltmarkt noch gewisse Absatzchancen ausrechneten. Doch auch jener immer größer werdende Teil der deutschen Wirtschaft, der auf die staatlichen Rüstungsaufträge fixiert war, hoffte darauf, daß durch das Abkommen von München »das Tempo der Aufrüstung verlangsamt und die Überbelastung der Wirtschaft auf ein erträgliches Maß zurückgeschraubt werden würde« (Thomas, 1966, S.130).

Die schon erwähnte Überbeanspruchung der Arbeitskräfte und der Maschinen ging sogar so weit, daß mehrere große Rüstungsfirmen sich im Sommer 1939 weigerten, weitere Rüstungsaufträge anzunehmen. Sie könnten die Ausführung nicht mehr garantieren (Mason, 1975 b, S. 171; Bagel-Bohlan, 1973, S. 130f.).

Der stärkste Gegner einer zu sehr forcierten Aufrüstungspolitik war seit 1936 der Reichswirtschaftsminister und Reichsbankpräsident Schacht. Er blieb zwar ein scharfer Gegner einer allgemeinen Abwertung der Reichsmark, wollte aber ansonsten den Versuch wagen, Deutschland wieder enger in den Weltmarkt einzubinden. Die Rückeinbindung in ein multilaterales Handelssystem forderte auch der Reichspreiskommissar Goerdeler. Er befürchtete, daß die Loslösung Deutschlands vom Weltmarkt zum Wirtschaftskrieg aller gegen alle führen müßte. In seinem letzten großen Gutachten vom 7. September 1936 versuchte er in einem dramatischen Appell, den wirtschaftspolitischen Kurs der Reichsregierung zu ändern. Seine Forderung lief darauf hinaus, ein westliches Bündnissystem zu aktualisieren, nicht zuletzt, um damit revisionistische Forderungen im Osten einlösen zu können (Doering, 1969, S.226f.). Die Handlungsalternativen, die hier angeboten wurden, hat. ten jedoch kaum Chancen, realisiert zu werden, sowohl aus ökonomischen (a) als auch aus herrschaftspolitischen (b) Gründen.

a) Eine Rückorientierung zum Weltmarkt war zwar an sich denkbar, wäre jedoch äußerst schwierig zu bewältigen gewesen, von der politischen Durchsetzbarkeit einmal ganz abgesehen. Mit einer Abwertung wäre es sicherlich nicht getan gewesen. Es darf dabei ja auch nicht übersehen werden, daß Deutschland durchaus Teilabwertungen gegenüber bestimmten Ländern - wenn auch in verdeckter Form - vorgenommen hatte und daß der Export subventioniert wurde. Schließlich sei auch noch darauf hingewiesen, daß durch eine Abwertung der Reichsmark zum einen die Auslandsschulden erhöht, zum anderen die Rohstoffimporte verteuert worden wären, und dies auf dem Hintergrund der Tatsache, daß sich die Terms of Trade seit 1934 verschlechterten.

Gravierender jedoch war, daß sich der Weltmarkt selbst - wie schon erwähnt - ungeachtet zeitweiser leichter Erholung äußerst krisenhaft, besonders wieder am Ende der dreißiger Jahre, entwickelt hatte. Von besonderer Bedeutung war ferner die Tatsache, daß die deutsche Industrie in weiten Teilen sich bereits zu sehr auf die bequemen staatlichen Rüstungsaufträge fixiert hat, folglich enorme Umstellungsschwierigkeiten hätte in Kauf nehmen müssen, wollte sie sich erneut auf den Weltmarktbedarf einrichten. Allenfalls hätte die ökonomische Lage des Kapitals durch eine massive Hilfe des Auslandes renormalisiert wer- 
den können, so die These von Sohn-Rethel. Ob das Ausland dazu überhaupt fähig und bereit gewesen wäre, durch massive Hilfe die ökonomische Kehrtwendung in Deutschland herbeizuführen, ist eine Frage, die sich im realen Ereignisablauf erst gar nicht stellte und insofern recht hypothetisch bleibt.

Um so mehr Gewicht bekommt deshalb die Frage, wie groß die Umstellungsschwierigkeiten für die deutsche Industrie gewesen wären, wobei für diese Frage vorübergehend die Verschlechterung der gesamten Weltmarktsituation außer acht bleiben muß. Eine solche Frage kann ansatzweise dadurch beantwortet werden, daß nach dem Stand der Arbeitsproduktivität gefragt wird. Nach bisherigen Kenntnissen waren trotz des Rationalisierungsschubs um 1924/25 die in Deutschland in den dreißiger Jahren angewandten Produktionsverfahren nicht auf dem modernsten Stand. Eine Automatisierung der Produktionsprozesse war noch nicht in großem Umfang durchgeführt. Eine Massenproduktion im eigentlichen Sinne existierte erst in Anfängen. Die Typenvielfalt und die mangelnde Normung der Einzelteilherstellung verteuerten die Gesamtproduktion (Bagel-Bohlan, 1973, S.139). In der Zeit zwischen 1933 und dem Kriegsausbruch war kein nennenswerter Zuwachs in der Arbeitsproduktivität zu verzeichnen (ebd., S. 137; Klein, 1959, S.71). Die Produktivität pro Beschäftigten in der Industrie der USA übertraf die der Deutschen um mehr als das Doppelte (in: Bagel-Bohlan, 1973, S.32). Die beträchtliche Steigerung der Arbeitsproduktivität in Deutschland während des Krieges zeigte die relative Rückständigkeit der deutschen Industrie vor Kriegsausbruch (Milward, 1977, S.75). Die militärische Führung beklagte häufig die handwerklich anmutende Vielfalt des Kriegsgeräts.

Die Entwicklung der Arbeitsproduktivität verlief freilich in den einzelnen Branchen sehr unterschiedlich. So war die Arbeitsproduktivität im Kohlebergbau in Deutschland höher als im übrigen Europa, mit Ausnahme Polens (!) und der Niederlande (Bagel-Bohlan, 1973, S.97f.). Dies wird durch Aussagen der damaligen Vertreter der Bergwerksunternehmen untermauert, die ebenfalls von einer hohen Technisierung des deutschen Bergbaus sprachen und zur Erklärung des Leistungsrückgangs auf andere Faktoren verwiesen, vor allem auf den Faktor Arbeitskraft sowie auf die Inbetriebnahme minderergiebiger Betriebspunkte, schließlich auf Schwierigkeiten bei der Beschaffung von Gerätschaften aus Eisen infolge des Materialmangels (Dt. Volkswirt 10.12.1937). Auch in der Eisen- und Stahlindustrie war für Rationalisierung und Mechanisierung »im Dienste der reinen Kostenersparnis und der Steigerung der reinen Arbeitsleistung je Mann ... mindestens in den ersten Gewinnungs- und Produktionsstufen nicht mehr sehr viel Spielraum vorhanden« (Dt. Volkswirt 9.9.1938). Allerdings wird zugegeben, daß Verfeinerung, Verbesserung und Spezialisierung der Erzeugnisse noch möglich seien und daß dies auch eine wichtige Aufgabe für die Zukunft darstelle, wobei vor allem an Stahllegierungen und feinere Walzprodukte gedacht wurde (Dt. Volkswirt, 9.9.1938). - Bei der Kaliindustrie stellte man 1938 eine Tendenz zu steigenden Investitionen fest, die vor allem der Rationalisierung der Produktion dienen sollten (ebd., 3.6.1938). Die Kostensenkungen ließen allerdings noch auf sich warten (ebd.). - Eine ähnliche Entwicklung ist uns von der Zementindustrie überliefert. Auch hier wurden Investitionserhöhungen vorgenommen, um die Rationalisierung in dieser Branche voranzutreiben, wobei auch hier die Früchte noch auf sich warten ließen (ebd.). Dagegen war in der Textilindustrie sogar ein Produktivitätsrückgang zu verzeichnen (Klein, 1959, S.71). Soweit Rationalisierungsbestrebungen im Gange waren, stießen diese auf Schwierigkeiten infolge des Engpasses bei Werkzeugmaschinen. Die deutsche Werkzeugmaschinenindustrie war offensichtlich völlig überfordert (Thomas, 1966, S. 240f.). Zwar war die Werkzeugma- 
schinenproduktion schon wesentlich angestiegen, doch waren hier aufgrund des Arbeitskräftemangels Grenzen gesetzt. Wegen des Devisenmangels stellte auch der Import ausländischer Werkzeugmaschinen keine Lösung der Probleme dar. Ein Vergleich mit den Vereinigten Staaten machte den Engpaß in dieser Branche besonders deutlich: Setzt man den Index der Werkzeugmaschinenproduktion in den USA 1939 mit 100 an, so erhöhte sich dieselbe dort bis zum Jahre 1942 auf 662, während die Vergleichszahlen für Deutschland 129 (1941) und 126 (1942) betrugen (Milward, 1977, S.77). Interessant ist in diesem Zusammenhang, daß in Deutschland 1945 nur $8 \%$ aller Werkzeugmaschinen Spezialwerkzeugmaschinen waren. Die Konzentration auf Universalwerkzeugmaschinen brachte zwar einerseits für die deutsche Produktion eine große Flexibilität mit sich, bedeutete jedoch eine Beschränkung bei der Standardisierung der Produktherstellung (ebd., S.78).

Im ïbrigen war die Leistungsfähigkeit der deutschen Werkzeugmaschinen nicht auf dem neuesten Stand. So wurde z.B. durch den ersten im Kriege erfolgten Einsatz von Hochleistungswerkzeugmaschinen die Bearbeitungszeit für Geschützrohre auf etwa ein Zehntel der ursprünglichen Bearbeitungszeit herabgesetzt (Bagel-Bohlan, 1973, S.201).

Zusammenfassend zeigt sich das Bild, daß die eindrucksvolle Produktionssteigerung der deutschen Industrie im allgemeinen weniger auf technologisch erhöhter Arbeitsproduktivität als vielmehr auf der Ausweitung der Produktionsanlagen einerseits und der Arbeitszeitausdehnung sowie der Arbeitsintensivierung andererseits beruht hat.

Die Regierung hatte die Einführung rationeller Produktionsmethoden durchaus gefördert. So wurden für die einzelnen Branchen »Kostenrichtsätze« aufgestellt und den Unternehmern erlaubt, Gewinne zu behalten, die sie durch Rationalisierung oder durch Erhöhung der Produlttion innerhalb der Richtsätze erzielten (Blaich, 1979, S. 47f.). Gerade als es darum ging, Arbeitskräfte einzusparen, hätte allein eine weitere Rationalisierung der Produlstion Abhilfe schaffen können. Daran mußte auch der NS-Regierung gelegen sein. Trotzdem war die Industrie vor dem Krieg nicht überaus stark an Rationalisierungsmaßnahmen interessiert. General Thomas meinte, daß vor dem Kriege nur diejenigen Betriebe sich einen entsprechend modernen Maschinenpark angeschafft hätten, die auf entsprechende Wehrmachtsaufträge verweisen konnten (1966, S.240f.). Die Abwendung vom Weltmarkt bedeutete Verzicht auf die Vorteile der internationalen Arbeitsteilung und den weitgehenden Wegfall des internationalen Wettbewerbs. Mit dem Rückgang des internationalen Warenaustausches verkümmerte der internationale Erfahrungsaustausch. Damit fielen auch Rationalisierungsanreize und -zwänge weg. Auch die in der Rüstungsindustrie üblichen Kostenerstattungspreise wirkten auf etwaige Rationalisierungsbestrebungen vielfach eher hemmend als fördernd. (Bagel-Bohlan, 1973, S. 141).

Abgesehen von der nicht-optimalen Entwicklung der Arbeitsproduktivität kamen noch andere Faktoren hinzu, die eine Rückbindung Deutschlands in den ohnehin schwachen Weltmarktzusammenhang erschwert hätten. Zu verweisen ist vor allem auf die gestiegenen Selbstkosten (z.B. Dt. Volkswirt, 11.3.1938).

Schließlich verminderte sich die Konkurrenzfähigkeit Deutschlands durch dessen Ausrichtung auf den relativ geschützten südosteuropäischen Markt, bei dem man sich preislich nicht anzustrengen brauchte.

Das deutsche Preisniveau lag insgesamt gesehen weit über dem der Auslandsmärkte. Die Exportfirmen waren deshalb auf staatliche Unterstïtzung zur künstlichen Senkung der deutschen Ausfuhrpreise auf ein wettbewerbsfähiges Niveau angewiesen. Doch waren die Erfolge letztendlich sehr begrenzt. Viele Betriebe hatten es einfach satt, sich dem mühsa- 
men, risikoreichen und staatlich dirigierten Exportbetrieb zuzuwenden, während doch die zunehmenden Staatsaufträge einfacher, lukrativer und risikoloser waren. Damit zerrissen aber auch alte Handelsbeziehungen. Insgesamt gesehen kann man deshalb durchaus von einer eingeschränkten Weltmarktfähigkeit der deutschen Industrie sprechen.

Eine Umstellung auf die so hart gewordenen Weltmarktbedingungen waren unter solchen Umständen kaum zu erwarten und hätten einen »unerträglich langen wirtschaftspoliti-

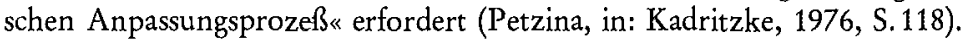

b) Auch aus herrschaftspolitischen Gründen waren die Alternativvorschläge nicht durchsetzbar. Jede massive Änderung der Wirtschaftspolitik in diese Richtung hätte den labilen Herrschafts- und Interessentrust von Industrie, Bürokratie, Militär und NSDAP zerstört. Dieser Herrschaftstrust war mittlerweile trotz großer Friktionen personell und strukturell sehr ineinander verwoben und vor allem über die forcierte Aufrüstungspolitik funktionell stark aufeinander bezogen, so daß trotz der labilen Strukturen ein Auseinanderbrechen von selbst kaum zu erwarten war. Ein Eingehen auf solche Alternativvorschläge hätte vor allem die alte Bürokratie wieder gestärkt und wäre zu Lasten der NS-Führung gegangen.

Um die »klassische« Auseinandersetzung über die Primatsthese Primat der Ökonomie (Czichon) versus Primat der Politik (Mason) - ist es mittlerweile ruhig geworden. Dies hängt zum einen damit zusammen, daß man sich nach einer stark theorie-orientierten Phase nunmehr der Empirie zuwendet, zum anderen scheint wohl ein Grund darin zu liegen, daß der Begriff »Primat« zu sehr an Oberflächenerscheinungen orientiert ist. Stattdessen wird - inspiriert durch bestimmte staatstheoretische Überlegungen (Blanke) - der durch die expansionsorientierte forcierte Aufrüstung gegebene funktionelle Zusammenhang von Ökonomie und Politik in den Mittelpunkt gestellt (Sohn-Rethel, Kadritzke), wodurch sowohl die relative Verselbständigung der Exekutive als auch deren Handlungsspielraum näher bestimmt und genauer begründet werden kann. Der »Trust«-Begriff ist deshalb wohl geeigneter als der »Primats«-Begriff, weil in ihm der krisenlösungsorientierte funktionelle Wirkungszusammenhang zum Ausdruck kommt. Er verdeutlicht den Wirkungs- und Interessenzusammenhang auch besser als die Vorstellung von den diversen Machtsäulen (Neumann), zu deren Charakterisierung einseitig auf die in den jeweiligen Bereichen relativ große Selbständigkeit der «Machtsäulen* (Militär, Bürokratie, Wirtschaft, Partei) hingewiesen wird.

2. Kam also aus ökonomischen und herrschaftspolitischen Gründen das erste Alternativkonzept nicht zum Zuge, so spielen herrschaftspolitische Gründe die entscheidende Rolle bei der Verhinderung der Durchführung eines zweiten damals auch schon entwickelten Alternativprogramms. Dieses wurde vom Chef der Wehrwirtschaftsorganisation, Oberst (später General) Thomas, vorgetragen. Er forderte die Sicherung und Steigerung der Rüstungsproduktion durch scharfe Einschränkungen auf allen anderen Gebieten. Vor allem sollten die Konsumbedürfnisse noch rigoroser als bisher gedrosselt werden. Thomas war schon vor dem Krieg der Ansicht, die er im November 1939 dann in die bekanntgewordenen Worte faßt:

"(...) mit Radioapparaten, Staubsaugern und Küchengeräten werden wir England nie besiegen können" (in: Mason, 1975 b, S.170).

Man war sich allgemein darüber damals schon im Klaren, daß eine Steigerung der Rüstungsproduktion nur noch möglich war, wenn es gelang, die zivilen Sektoren zu beschneiden. Doch das war eben politisch äußerst schwierig durchzusetzen, denn die Massen erwarteten immer mehr, auch einmal wenigstens etwas an der Hochkonjunktur teilhaben zu können. Zweifellos geriet die Regierung dadurch unter Handlungsdruck, wie auch Herbst (1978, 
S.374f.) feststellt. Das Rezept von Thomas hätte überdies die Einführung strenger Kontrollen auf dem Arbeitsmarkt und in der Lohn- und Preisbildung erfordert. Außerdem wären erhebliche Steuererhöhungen und eine viel konsequentere Bewirtschaftung der vorhandenen Kapazitäten und Rohstoffe, als dies tatsächlich der Fall war, nötig gewesen.

Der Zwangscharakter des Staates hätte dadurch eine neue Dimension erhalten. Und dies wäre aus herrschaftspolitischen Gründen - wegen der großen Instabilität des politischen Systems - nur mit äußerst großem Risiko durchzusetzen gewesen.

Der häufig vorgebrachte Hinweis auf die 'Tatsache, daß Deutschland in der sogenannten Ära Speer sein Rüstungspotential noch beträchtlich steigern konnte, läßt sich nicht auf die Vorkriegszeit übertragen. Was vor Kriegsausbruch aus ökonomischen und herrschaftspolitischen Gründen wohl nicht mehr zu realisieren war, ließ sich durchaus während des Krieges verwirklichen, nicht zuletzt durch die kolossale Ausbeutung großer Regionen und Vö1ker und nicht zuletzt deshalb, weil viele Deutsche während des Krieges höhere Anstrengungen vollbrachten - aus dem Bewußtsein heraus, daß es nun ums Ganze ginge und daß sie alle im gleichen Boot säßen.

3. Die dritte "Lösungsmöglichkeit« lag in der (gemessen an der Planung) verfrühten Kriegsentfesselung. Hitler visierte bekanntlich noch im November 1937 den Krieg erst für das Jahr 1943 an. Auch war im »Wehrwirtschaftlichen Neuen Erzeugungsplan« vom Juli 1938 als Zieljahr das Jahr 1942/43 angegeben. Doch die Zeit schien immer mehr zu drängen. Hitler und Goering wußten offensichtlich sehr genau, daß man die forcierte Aufrüstung angesichts der schwachen ökonomischen Ressourcen nicht lange mehr werde durchhalten können (Herbst, 1978, S.381). In der Ansprache vor den Befehlshabern vom 22.8.1939 begründete Hitler die Notwendiglseit des Kriegsbeginns mit den bedeutungsvollen Worten:

"Wir haben nichts zu verlieren, wohl zu gewinnen. Unsere wirtschaftliche Lage ist infolge unserer Einschränkungen so, daß wir nur noch wenige Jahre durchhalten können. Göring kann das bestätigen. Uns bleibt nichts anderes übrig, wir müissen handeln« (in: ebd., S. 328) ${ }^{8}$

Nach einer zweiten Version soll Hitler bei dieser Gelegenheit gesagt haben:

"Göring hat ja ausgeführt, daß Vierjahresplan gescheitert und wir am Ende, wenn nicht Sieg im kommenden Krieg" (in: Mason, 1975 b, S. 184).

Auch in der Oktoberdenkschrift von 1939 meinte Hitler, die militärische Auswertung der Deutschen Volkskraft sei

sin einem Ausmaß vorhanden, das in kurzer Zeit jedenfalls durch keinerlei Anstrengungen wesentlich verbessert werden« könne (in: Volkmann, 1976, S. 56).

Die Gefahr, den Rüstungsvorsprung gegenüber dem Ausland zu verlieren, war nicht von der Hand zu weisen. Deshalb entschied sich Hitler, den damals noch vorhandenen relativen Rüstungsvorsprung für eine territoriale Expansionspolitik auszunutzen (vgl. Volkmann, 1976, S.56f.; Geyer, 1980, S.449). Der Herbst 1939 war nämlich rüstungsökonomisch gesehen für eine militärische Expansion der optimale Zeitpunkt, »soweit man in der skizzierten Krisensituation von einem Optimum überhaupt sprechen « kann, denn das Jahr 1939 bildete einen Kulminationspunkt hinsichtlich der Höhe der erreichbaren Industrieproduktion in "Friedenszeiten " (ebd., S. 57; näheres: Deist, 1979, S. 447). ${ }^{10}$

Trotz dieser hohen Industrieproduktion und trotz der relativ erfolgreichen Rüstungsanstrengungen war man absolut gesehen für einen Krieg schlecht gerüstet. Vor allem fehlte es an der sogenanten Tiefenrüstung (Nachschubbereitung, Geräteersatz, Verbesserung der In- 
dustriekapazitäten etc.), sehr zum Leidwesen des Chefs der Wehrwirtschaftsorganisation, Thomas. Die Rüstung reichte allerdings aus, um eine ganz bestimmte Art Krieg zu führen (und »natürlich« zu gewinnen), nämlich den sogenannten Blitzkrieg (dazu Milward, 1975; Blaich, 1979, S.35). Man wollte halb Europa ausplündern, um dadurch immer weitere Expansionen möglich zu machen.

Sicherlich ist die Entwicklung hin zum Ausbruch kriegerischer Expansion nicht als die Folge einer von der Komplexität historisch-konkreter Entwicklungsabläufe zu abstrahierende Automatik zu begreifen. Vor allem ist zu berïcksichtigen, daß im Unterschied zur Großen Krise von 1929ff. die Gesamtkrise von 1938/39 noch nicht durchgängig an die Oberfläche und damit in das Bewußtsein der breiten Bevölkerungsschichten gekommen ist. Doch wurde eine solche offene Gesamtkrise für die nahe Zukunft von der NS-Führungsspitze antizipiert. Und wie unsere Darlegungen zeigen, wohl auch zu Recht. Wenngleich also keine absolute Zwangsläufigkeit im Spiele war, so war der Zeitpunkt des Kriegsausbruchs doch die wahrscheinliche Folge der stark angewachsenen Widersprüche zwischen »kriegerischen Absichten, ökonomischen Möglichkeiten und verstärktem sozialem Druck « (Petzina, 1975, S.80).

$\mathrm{Da}$ diese Widersprüche bereits in der Geburtsstunde des Dritten Reiches angelegt waren sowohl in ökonomischer als auch in sozio-politischer Hinsicht - ja sozusagen zu den Konstitutionsbedingungen des deutschen Faschismus gehörten, ist es in der Tat sinnvoll und auch wohl notwendig, trotz unbestreitbarer Wirtschaftswunderphänomene und Stabilitätsmomente im Herrschaftsgefüge von einer hohen Instabilität, ja sogar von einer »Krise in Permanenz« (Peukert, 1982, S. 47ff.) zu sprechen. 


\section{Anmerkungen}

○ Zum Krisenbegriff vgl. den Überblick bei Jännicke, 1973; in historischer Perspektive: Vierhaus, 1978.

1 Die Landwirtschaft hatte allerdings auch mit anderen Krisenphänomenen zu kämpfen. Vor allem ist hier die Preisschere zwischen landwirtschaftlichen und industriellen Produkten zu nennen.

2 Es fehlen entsprechende firmenbezogene Untersuchungen. Eine Ausnahme stellt die Arbeit von Seebold über den Bochumer Verein dar. Auch Seebold spricht von einem »erhöhten Verschleiß der Betriebsanlagen«, von einer »angespannten Auftragslage« (trotz Rückgang des Exportanteils), sodaß es zu einer Verlängerung der Lieferfristen kam. Im Zeitraum von 1936/37 bis 1938/39 stieg der Gesamt umsatz des Bochumer Vereins von 176 Mio RM auf 233 Mio RM. Allein die Rohstahlerzeugung erhöhte sich im gleichen Zeitraum um 32 v.H. Die Kapazitätsgrenze wurde damit erreicht (Seebold, 1981, S.93f., 97).

3 Der deutsche Werkzeugmaschinenbau nahm von 1933-1938 um 281\% zu (Bagel-Bohlan, 1973, S.27).

4 Entwicklung der Industrie-Investitionen (preisbereinigt, $1928=100$ )

$1928 \quad 1936 \quad 1939$

Produlstionsgüterindustrie $\quad 100 \quad 116 \quad 256$

Konsumgüterindustrie $\quad 100 \quad 80 \quad 119$

(Aus: Petzina, 1975, S.80.)

5 Während sich das Bruttosozialprodukt von 1932-1939 in etwa verdoppelte, stiegen die Rüstungskosten im selben Zeitraum um 35\% (Dülffer, 1976, S.451).

6 Sohn-Rethel führt aus, daß die Aufrüstungsgüter nicht reproduzierbare Werte darstellten, d.h., daß sie weder der Erhaltung menschlicher Arbeitskraft dienten, noch in materielle Produktionsmittel eingingen. Boelcke betont dagegen vor allem die disproportionalen Entwicklungen der Volkswirtschaft sowie die Finanzschwierigkeiten als Folge der forcierten Aufrüstungspolitik (1975, S.19).

7 Die Lohnneubemessung gedieh am weitesten in der Eisen- und Metallindustrie.

8 Herbst interpretiert mißlich, wenn er einseitig die zwei Worte »wenige Jahre « betont und deshalb diesen Ausspruch Hitlers als Zeichen defizienten Krisenbewußtseins ansieht (1978, S. 382).

9 Volkmann bezieht mit Recht diese Aussage vor allem auf den damals geplanten Überfall auf Luxemburg und Belgien als ökonomisches Reserveland. Der Ausspruch ist jedoch auch für die Beurteilung der Gesamtsituation durch Hitler wichtig (1976, S.56).

10 Petzina meint allerdings: »Der Stand der wirtschafts- und rüstungspolitischen Mobilisierung durch den Vierjahresplan wie auch die nach wie vor relativ große Auslandsabhängigkeit bei strategischen Rohstoffen hätten für einen späteren Zeitpunkt gesprochen.« (1975, S. 80).

\section{Literaturverzeichnis}

Bagel-Bohlan, Elke Anja, Die industrielle Kriegsvorbereitung in Deutschland von 1936 bis 1939, Diss., Witterschlick 1973

Blaich, Fritz, Wirtschaft und Rüstung in Deutschland 1933-1939, in: Wolfgang Benz, Hermann Graml (Hg.), Sommer 1939. Die Großmächte und der europäische Krieg, Stuttgart 1979

Blanke, Bernhard/Jürgen, Ulrich/Kastendiek, Hans, Zur neueren marxistischen Diskussion über die Analyse von Form und Funktion des bürgerlicben Staates. Überlegungen zum Verhältnis von Politik und Ökonomie, in: Prokla, H.14/15, 1975

Blanke, Bernhard, Formen und Wandel des politischen Systems in der bürgerlichen Gesellschaft, in: Staat (= Politische Ökonomie: Handbuch 5), Frankfurt 1977 
Boelcke, Willi, A., Probleme der Finanzierung von Militärausgaben, in: Friedrich Fortmeier/HansErich Volkmann, Wirtschaft und Rüstung am Vorabend des Zweiten Weltkrieges, Düsseldorf 1975 Boelcke, Willi, A., Kriegsfinanzierung im internationalen Vergleich, in: Forstmeier, Friedrich/Volkmann, Hans-Erich, Kriegswirtschaft und Rüstung 1939 bis 1945, Düsseldorf 1977

Boelcke, Willi, A., Zur internationalen Goldpolitik des NS.Staates. Ein Beitrag zur deutschen Wäbrungsund Außenwirtschaftspolitik 1933 bis 1945, in: Manfred Funke (Hg.), Deutschland und die Mächte. Materialien zur Außenpolitik des Dritten Reiches, Düsseldorf 1978 (2. Aufl.)

Bacher, Karl-Dietrich, Auf dem Wege zum 20. Juli 1944, in: Löwenthal, Richard/von zur Mühlen, Patrik (Hg.), Widerstand und Verweigerung in Deutschland 1933 bis 1945, Berlin-Bonn 1982

Broszat, Martin, Der Staat Hitlers, München 1969

Carroll, Berenice A., Design for Total War. Arms and Economics in the Third Reich, the Hague 1968

Czichon, Eberhard, Der Primat der Industrie im Kartell der nationalsozialistischen Macht, in: Das Argument 47, 1968.

Deist, Wilhelm u.a., Ursachen und Voraussetzungen der deutschen Kriegspolitik ( $=$ Das Deutsche Reich und der Zweite Weltkrieg Bd.1), Stuttgart 1979

Der deutsche Volkswirt, verschiedene Nummern der Jahre 1938/1939

Doering, Dörte, Deutsche Außenwirtschaftspolitik 1933-1935. Die Gleichschaltung der Außenwirtschaft in der Frïhphase des nationalsozialistischen Regimes, Diss., Berlin 1969

Dülffer, Jost, Der Beginn des Krieges 1939; Hitler, die innere Krise und das Mächtesystem, in: Geschichte und Gesellschaft, 2.Jg. 1976, H.4

Elfferding, Wieland, Opferritual und Volksgerneinschaftsdiskurs am Beispiel des Winterbilfswerks, in: Fa* schismus und Ideologie, Bd. 2 (= Argument Sonderband 62), Berlin 1980

Erbe, René, Die nationalsozialistische Wirtschaftspolitik 1933 bis 1939 im Lichte der modernen Theorie, Zürich 1958

Fischer, Wolfram, Deutsche Wirtschaftspolitik 1918-1945, Obladen 1968 (3. Aufl.)

Fischer, Wolfram, Probleme von Außenhandel und Aufrüstung, in: Friedrich Fortmeier/Hans-Erich Volkmann ( $\mathrm{Hg}$.$) , Wirtschaft und Rüstung am Vorabend des Zweiten Weltkrieges, Düsseldorf 1975$

Forstmeier, Friedrich/Volkmann, Hans-Erich (Hg.), Wirtschaft und Rüstung am Vorabend des Zweiten Weltkrieges, Düsseldorf 1975

Forstmeier, Friedrich/Volkmann, Hans-Erich (Hg.), Kriegswirtschafi und Rüstung 1939 bis 1945, Düsseldorf 1977

Friemert, Chup, Die Organisation des Ideologischen als betriebliche Praxis, in: Faschismus und Ideologie, Bd. 2 ( = Argument Sonderband 62), Berlin 1980

Gamm, Hans-Jochen, Der Flüsterwitz im Dritten Reich, München 1980 (2. Aufl.)

Geyer, Michael, Aufrüstung oder Sicherbeit. Die Reichswebr in der Krise der Machtpolitik 1924-1936 (=-Veröffentlichungen des Instituts für europäische Geschichte, Mainz, Bd. 91), Wiesbaden 1980

Hennig, Eike, Industrie, Aufrüstung und Kriegsvorbereitung im deutschen Faschismus. Anmerkungen zum Stand der neweren Faschismusdiskussion, in: Friedrich Forstmeier/Hans-Erich Volkmann $(\mathrm{Hg}$ ), Wirtschaft und Rüstung am Vorabend des Zweiten Weltkrieges, Düsseldorf 1975

Herbst, Ludolph, Die Krise des nationalsozialistischen Regimes am Vorabend des Zweiten Weltkrieges und die forcierte Aufrüstung, in: Vierteljahreshefte für Zeitgeschichte 26, 1978, Nr, 3

Herbst, Ludolph, Die Mobilmachung der Wirtschaft 1938/39 als Problem des nationalsozialistischen Herrschaftssystems, in: Wolfgang Benz/Hermann Graml (Hg.), Sommer 1939. Die Großmächte und der europäische Krieg, Stuttgart 1979

Herbst, Ludolph, Der Totale Krieg und die Ordnung der Wirtschaft. Die Kriegswirtschaft im Spannungsfeld von Politik, Ideologie und Propaganda 1939 bis 1945, Stuttgart 1982

Jacobeit, Sigrid, Zum Alltag der Bäuerinnen in Klein-und Mittelbetrieben wäbrend der Zeit des deut. schen Faschismus 1933 bis 1939, in: Jahrbuch für Wirtschaftsgeschichte 1982/1

Jänicke, Martin (Hg.), Herrschaft und Krise, Beiträge zur politikwissenschaftlichen Krisenforschung, Opladen 1973

Kadritzke, Niels, Faschismus und Krise. Zum Verbältnis von Politik und Ökonomie im Nationalsozialis- 
mus, Frankfurt 1976

Kershaw, Jan, Der Hitler-Mythos. Volksmeinung und Propaganda im Dritten Reich, Stuttgart 1980

Klein, Burton H., Germany's Economic Preparations for War, Cambridge/Mass. 1959

Kleßmann, Christoph/Pingel, Falk (Hg.), Gegner des Nationalsozialismus. Wissenschaftler und Widerstandskämpfer auf der Suche nach bistorischer Wirklichkeit, Frankfurt 1980

Kroll, Gerhard, Von der Weltwirtschaftskrise zur Staatskonjunktur, Berlin 1958

Löwenthal, Richard/von zur Mühlen, Patrik (Hg.), Widerstand und Verweigerung in Deutschland 1933 bis 1945, Berlin, Bonn 1982

Mason, Timothy, W., Der Primat der Politik - Politik und Wirtschaft im Nationalsozialismus, in: Das Argument 41, 1968

Mason, Timothy, W., Arbeiterklasse und Volksgemeinschaft. Dokumente und Materialien zur deutschen Arbeiterpolitik 1936-1939, Obladen 1975a

Mason, Timothy, W., Innere Krise und Angriffskrieg 1938/39, in: Friedrich Forstmeier/Hans-Erich Volkmann (Hg.), Wirtschaft und Rüstung am Vorabend des Zweiten Weltkrieges, Düsseldorf 1975b

Mason, Timothy, W., Die Bändigung der Arbeiterklasse im nationalsozialistischen Deutschland, in: Carola Sachse u.a., Angst, Belobnung, Zucht und Ordnung. Herrschaftsmechanismen im Nationalsozialismus, Opladen 1982

Milward, Alan, S., Der Einfluß ökonomischer und nicht-ökonomischer Faktoren auf die Strategie des Blitzkriegs, in: Friedrich Forstmeier/Hans-Erich Volkmann ( $\mathrm{Hg}_{\text {. }}$, Wirtschaft und Rüstung am Vorabend des Zweiten Weltkrieges, Dïsseldorf 1975

Milward, Alan, S., Arbeitspolitik tnd Produktivität in der deutschen Kriegswirtschaft unter vergleichendem Aspekt, in: Friedrich Forstmeier/Hans-Erich Volkmann (Hg.), Kriegswirtschaft und Rüstung 1939 bis 1945, Düsseldorf 1977

Milward, Alan, S., Der Zweite Weltkrieg, München 1977

Neumann, Franz, Bebemoth. Struktur und Praxis des Nationalsozialismus 1933 bis 1934, Nachwort und neu herausgegeben von Gerd Schäfer, Frankfurt 1977

Peukert, Detlev, Volksgenossen und Gemeinschaftsfremde. Anpassung, Ausmerze und Aufbegebren unter dem Nationalsozialismus, Köln 1982

Petzina, Dieter, Autarkiepolitik im Dritten Reich. Der nationalsozialistische Vierjabresplan, Stuttgart 1968

Petzina, Dietmar, Grundrißder deutschen Wirtschaftsgeschichte 1918 bis 1945, in: Deutsche Geschichte seit dem Ersten Weltkrieg, Bd. II, Stuttgart 1973

Petzina, Dietmar, Vierjabresplan und Rüstungspolitik, in: Friedrich Forstmeier/Hans-Erich Volkmann ( $\mathrm{Hg}$.$) , Wirtschaft und Rüstung am Vorabend des Zweiten Weltkrieges, Düsseldorf 1975$.

Petzina, Dietmar, Die deutsche Wirtschaft in der Zwischenkriegszeit, Wiesbaden 1977

Radkau, Joachim, Entscheidungsprozesse und Entscheidungsdefizite in der deutschen Außenwirtschaftspolitik 1933 bis 1940, in: Geschichte und Gesellschaft 2, 1976, H. 1

v. Saldern, Adelheid, Mittelstand im "Dritten Reichw. Handwerker - Einzelbändler - Bauern, Frankfurt 1979

Schoenbaum, David, Die braune Revolution. Eine Sozialgeschichte des Dritten Reiches, Köln, Berlin 1968

Schweitzer, Arthur, Big Business in the Third Reich, Bloomington 1964

Seebold, Gustav-Hermann, Ein Stablkonzern im Dritten Reich. Der Bochumer Verein 1927.1945, Wuppertal 1981

Siegel, Tilla, Lobnpolitik im nationalsozialistischen Deutschland, in: Carola Sachse u.a., Angst, Belobnung Zucht und Ordnung. Herrschaftsmechanismen im Nationalsozialismus (= Schriften des Zentralinstituts für sozialwissenschaftliche Forschung der Freien Universität Berlin, Bd. 41) Opladen 1982

Spode, Hasso, "Der deutsche Arbeiter reist «: Massentourismus im Dritten Reich, in: Gerhard Huck (Hg.), Sozialgeschichte der Freizeit. Untersuchungen zum Wandel der Alltagskultur in Deutschland, Wuppertal 1980 
Sohn-Rethel, Alfred, Ökonomie und Klassenstruktur des deutschen Faschismus, Frankfurt 1973

Sternberg, Fritz, Kapitalismus und Sozialismus vor dem Weltgericht, Hamburg 1951

Thomas, Georg, Geschichte der deutschen Wehr. und Rüstungswirtschaft (1918 bis 1943/45), hrsg. v. Wolfgang Birkenfeld, Boppard/Rhein 1966

Vierhaus, Rudolf, Zum Problem bistorischer Krisen, in: Faber, Karl-Georg (Hg.), Historische Prozesse, München 1978

Volkmann, Hans-Erich, Außenbandel und Aufrüstung in Deutschland 1933 bis 1939, in: Friedrich Forstmeier/Hans-Erich Volkmann, Wirtschaft und Rüstung am Vorabend des Zweiten Weltkrieges, Düsseldorf 1975

Volkmann, Hans-Erich, Autarkie, Großraumwirtschaft und Aggression. Zur ökonomischen Motivation der Besetzung Luxemburgs, Belgiens und der Niederlande 1940, in: Militärgeschichtliche Mitteilun* gen 19, 1976, H. 1

Volkmann, Hans-Erich, NS-Außenhandel im sgeschlossenen Kriegswirtschaftsraum (1939 bis 1941), in: Friedrich Forstmeier/Hans-Erich Volkmann (Hg.), Kriegswirtschaft und Rüstung 1933 bis 1945, Düsseldorf 1977

Volkmann, Hans-Erich, Politik, Wirtschaft und Aufrüstung unter dem Nationalsozialismus, in: Manfred Funke (Hg.), Hitler, Deutschland und die Mächte. Materialien zur Außenpolitik des Dritten Reiches, Düsseldorf 1978 (2. Aufl.)

Volkmann, Hans-Erich, Die NS. Wirtschaft in Vorbereitung des Krieges, in: Wilhelm Deist u.a., Ursachen und Voraussetzungen der deutschen Kriegspolitik ( = Das Deutsche Reich und der Zweite Weltkrieg, Bd. 1), Stuttgart, 1979

Wagenführ, Rolf, Die deutsche Industrie im Kriege 1933 bis 1945, Berlin 1963 (2. Aufl.)

Wirtschaft und Statistik, hrsg. v. Statistischen Reichsamt 1938/1939

Wette, Wolfram, Ideologien, Propaganda und Innenpolitik als Voraussetzungen der Kriegspolitik des Dritten Reiches, in: Deist, Wilhelm u.a., Ursachen und Voraussetzungen der deutschen Kriegspolitik ( = Das Deutsche Reich und der Zweite Weltkrieg, Bd. 1), Stuttgart 1979 